\title{
RESEARCH
}

Open Access

\section{Antibiotic prescriptions in acute otitis media and pharyngitis in Italian pediatric outpatients}

\author{
E. Barbieri ${ }^{1 *}$ (D, D. Donà ${ }^{2,3}$, A. Cantarutti ${ }^{4,5}$, R. Lundin ${ }^{3}$, A. Scamarcia 6 , G. Corrao ${ }^{4,5}$, L. Cantarutti ${ }^{6}$ and \\ C. Giaquinto $2,3,4,6$
}

\begin{abstract}
Background: Acute otitis media (AOM) and pharyngitis are very common infections in children and adolescents. Italy is one of the European countries with the highest rate of antibiotic prescriptions. The aim of this study is to describe first-line treatment approaches for AOM and pharyngitis in primary care settings in Italy over six years, including the prevalence of 'wait and see' for AOM, where prescription of antibiotics is delayed $48 \mathrm{~h}$ from presentation, and differences in prescribing for pharyngitis when diagnostic tests are used.
\end{abstract}

Methods: The study is a secondary data analysis using Pedianet, a database including data at outpatient level from children aged 0-14 in Italy. Prescriptions per antibiotic group, per age group and per calendar year were described as percentages. "Wait and see" approach rate was described for AOM and pharyngitis prescriptions were further grouped according to the diagnostic test performed and test results.

Results: We identified 120,338 children followed by 125 family pediatricians between January 2010 and December 2015 for a total of 923,780 person-years of follow-up. Among them 30,394 (mean age 44 months) had at least one AOM diagnosis $(n=54,943)$ and 52,341 (mean age 5 years) had at least one pharyngitis diagnosis $(n=126,098)$. $82.5 \%$ of AOM diagnoses were treated with an antibiotic within $48 \mathrm{~h}$ (mainly amoxicillin and amoxicillin/clavulanate) and the "wait and see" approach was adopted only in $17.5 \%$ of cases. The trend over time shows an increase in broad spectrum antibiotic prescriptions in the last year (2015). 79,620 (63\%) cases of pharyngitis were treated and among GABHS pharyngitis confirmed by rapid test $56 \%$ were treated with amoxicillin. The ones not test confirmed were treated mainly with broad spectrum antibiotics.

Conclusions: Despite guidance to use the 'wait and see' approach in the age group analyzed, this strategy is not often used for AOM, as previously noted in other studies in hospital settings. Broad-spectrum antibiotic prescription was more frequent when pharyngitis was not confirmed by rapid test, in keeping with evidence from other studies that diagnostic uncertainty leads to overuse of antibiotics.

Keywords: "Antibiotic prescriptions", "wait and see", "acute otitis media" "pharyngitis", "Italian study", "Pediatric patient", "Pharmacoepidemiology"

\footnotetext{
* Correspondence: elisa.barbieri.5@phd.unipd.it

${ }^{1}$ Department for Woman and Child Health, University of Padua, Padua, Italy

Full list of author information is available at the end of the article
}

(c) The Author(s). 2019 Open Access This article is distributed under the terms of the Creative Commons Attribution 4.0 International License (http://creativecommons.org/licenses/by/4.0/), which permits unrestricted use, distribution, and reproduction in any medium, provided you give appropriate credit to the original author(s) and the source, provide a link to the Creative Commons license, and indicate if changes were made. The Creative Commons Public Domain Dedication waiver (http://creativecommons.org/publicdomain/zero/1.0/) applies to the data made available in this article, unless otherwise stated. 


\section{Background}

Antimicrobials are the most widely prescribed drugs in children worldwide, both in hospital [1,2] and community settings [3], especially at preschool age [4]. It has been estimated that $37-61 \%$ of hospitalized infants and children receive antibiotics [5-9] and almost half of antibiotic prescriptions in children are unnecessary [10-12]. Despite national and international efforts to promote appropriate antibiotic prescribing [13], Italy is one of the European countries with the highest rate of inappropriate antibiotic prescriptions (e.g. first line treatment prescription not in line with the guidelines or antibiotics prescribed for a diagnosis with a viral etiology), with an overuse of broad spectrum antibiotics [14-17].

Acute otitis media (AOM) and pharyngitis are two of the most common infections in pediatrics, and a main cause of antibiotic prescriptions [18].

$\mathrm{AOM}$ is an acute inflammation of the middle ear caused by viral (such as respiratory syncytial virus, rhinovirus, influenza viruses, and adenoviruses) or bacterial (such as Streptococcus pneumoniae, non-typeable Haemophilus influenzae, and Moraxella catarrhalis) infections [19]. AOM incidence in pediatric patients in Italy is estimated to be $16.8 \%$ [20] compared to worldwide incidence estimate of $10.85 \%$ [21].

National and international guidelines differentiate AOM treatment based on symptoms and the child's age. The Italian society of Pediatrics ("Società Italiana di Pediatria" - SIP) [22] and the Italian Federation of Pediatricians ("Federazione Italiana Medici Pediatri" - FIMP) [23] guidelines support the "wait and see" approach when appropriate, and when antibiotic choice is recommended, amoxicillin is designed as the first drug of choice. Antibiotic therapy should be immediately administered if the child is less than 6 months of age, while it could be delayed in older children.

Acute pharyngitis is also a very common pediatric diagnosis, and Group A $\beta$-hemolytic streptococcus (GABHS) causes $37 \%$ of cases of pharyngitis in children older than 3 years [24, 25]. Indeed, despite the percentage of GABHS pharyngitis is around $25-30 \%$, the antibiotic prescription rate appear to be from 10 to $20 \%$ higher $[26,27]$.

According to international guidelines [28-31] and the national family pediatricians consensus document [23], first line treatment for GABHS pharyngitis is either amoxicillin or penicillin $\mathrm{V}$ since GABHS remains universally susceptible to penicillins. The rapid diagnostic test is highly recommended for all the children with clinical symptoms of GABHS pharyngitis [30] since its high sensitivity and specificity had been proved in various studies [32].

In Italy, pediatricians (FP) follow children aged 0-6 at which point families choose whether an FP or general practitioner (GP) follows the child to 14 years of age.
Considering the high prevalence of these infections in the pediatric population, the aim of this study is to describe the first-line treatment approach for $\mathrm{AOM}$ and pharyngitis at primary care level in Italian children across different age groups and calendar time, considering the 'wait and see' approach for AOM and the use and results of rapid testing for pharyngitis.

\section{Methods}

\section{Study design}

This observational, retrospective, outpatient study used an established Italian network of FPs (Pedianet) from January 2010 to December 2015 for the assessment of first line treatment of $\mathrm{AOM}$ and pharyngitis.

\section{Data source}

Pedianet (http://www.pedianet.it), a pediatric general practice research database, contains reason for accessing healthcare, health status (according to the Guidelines of Health Supervision of the American Academy of Pediatrics), demographic data, diagnosis and clinical details (free text or coded using the 9th International Statistical Classification of Diseases and Related Health Problems system- ICD-9 CM), prescriptions (pharmaceutical prescriptions identified by the AnatomicalTherapeutical-Chemical code), specialist appointments, diagnostic procedures, hospital admissions, growth parameters and outcome data of the children habitually seen by about 125 family pediatricians (FPs) distributed throughout Italy. The FPs participation in the database is voluntary and patients and their parents provided consent for use of their data for research purposes. In Italy each child is assigned to a FP, who is the referral for any health visit or any drug prescription, thus the database contains a very detailed personal medical history. The data, generated during routine patient care using common software (JuniorBit ${ }^{\circ}$ ), are anonymized and sent monthly to a centralized database in Padua for validation. For this study data relating to 120,338 children, including information related to 132,667 diagnoses and 1,595,842 drug prescriptions, from 12 Italian regions (Friuli-Venezia Giulia, Liguria, Lombardia, Piemonte, Veneto, Abruzzo, Lazio, Marche, Toscana, Campania, Sardegna, and Sicilia) were considered.

The study and the access to the database were approved by the Internal Scientific Committee.

\section{Study population and case identification}

Information about patient characteristics, diagnosis, prescriptions, and test usage as well as rapid test results (positive, negative, and dubious) were obtained from the database for all identified cases of AOM or pharyngitis.

The study population included children aged from 0 months to 14 years with a primary ICD-9 code or 
descriptive diagnosis of acute otitis media (ICD-9-CM: 381.0, 381.00, 382, 382.0, 382.00 - "otite media acuta"), pharyngitis (034.0, 462- "faringotonsillite"), or tonsillitis (463- "tonsillite"). In order to avoid duplicates, medical records with the same diagnosis less than 30 days apart were considered as follow up of the initial case.

Pharyngitis diagnoses were divided in three groups based on FP diagnosis: GABHS pharyngitis, non-GABHS pharyngitis and non-defined pharyngitis. Strep-A rapid test was considered a suitable test to identify pharyngitis bacterial etiology. The test result was considered the gold standard for the diagnosis and all cases with a positive test result were classified as GABHS diagnosis, whereas negative results were classified as non-GABHS cases. Cases with dubious test results were classified according to the primary diagnosis.

Specific AOM exclusion criteria were: concomitant bacterial infections, ongoing antibiotic therapy, immunodeficiency or immunosuppressive therapy, tympanostomy tubes at the time of diagnosis, craniofacial abnormalities, chronic otitis media (381.1, 381.2, 381.3, $382.1,382.2,382.3)$, AOM complicated by mastoiditis (383), effusive otitis media, and chronic diseases (including cystic fibrosis and diabetes).

Specific pharyngitis exclusion criteria were: concomitant bacterial infections, ongoing antibiotic therapy, immunodeficiency or immunosuppressive therapy, previous tonsillectomy (28.2), chronic pharyngitis (472.1), chronic diseases (including cystic fibrosis and diabetes).

Ongoing antibiotic therapy was defined as antibiotic prescription in the 14 days before AOM or pharyngitis case.

For patients included in the study, only the first prescription per diagnosis was included.

\section{Statistical analysis}

\section{AOM statistical analysis}

Prescription distribution was described as the percentage of prescriptions per drug class (amoxicillin, amoxicillin and clavulanic acid (CV-Amoxicillin), II generation cephalosporins, III generation cephalosporins, macrolides, and other antibiotics).

We then calculated the frequency of prescriptions in each class according to age group ( $\leq 6$ months, 6-24 months, and $>24$ months, age bands used for treatment guidelines [23]) and trends in the prescription of antibiotics over time.

The "wait and see" approach was defined as all patients with AOM who did not receive antibiotic prescription within the first $48 \mathrm{~h}$ after diagnosis [33].

\section{Pharyngitis statistical analysis}

Pharyngitis diagnoses were described according to (i) age ( $<3$ years, $\geq 3$ years, age used for treatment guidelines [23]), (ii) test used to identify bacterial etiology, and (iii) results of the test. The frequency of prescriptions in each drug class was calculated by each of these strata as well.

\section{Results}

We identified 120,338 children followed by 125 family pediatricians participating in Pedianet between 2010 and 2015. Among them 30,394 had at least one AOM diagnosis and 52,341 at least one pharyngitis diagnosis.

\section{AOM population}

Among 30,394 children with AOM we recorded 54,943 distinct AOM diagnoses.

The mean age at diagnosis was 44 months, with $2 \%$ of children less than 6 months of age, 22\% between 6 and 24 months and $76 \%$ older than 24 months of age.

In 45,320 (82.5\%) AOM diagnoses the pediatrician prescribed an antibiotic within the $48 \mathrm{~h}$ from the diagnosis, while for 9623 cases (17.5\%) the 'wait and see' approach was preferred. In 535 AOM initially treated with the 'wait and see' approach (535/9623, 5.6\%), an antibiotic prescription was filled between 48 and $120 \mathrm{~h}$ after the diagnosis.

Amoxicillin and CV-Amoxicillin were the most frequently prescribed antibiotics overall (15,906/45320$35.1 \%$ vs $14,865 / 45320-32.8 \%$ ), followed by III generation cephalosporins (9114/45320). Macrolides were less prescribed with a frequency of $3 \%(1347 / 45320)$ (Table 1).

The distribution of first line antibiotic therapy according to different age groups is described in Fig. 1 and Table 1. The use of amoxicillin seems to decrease with increasing age, while cephalosporins and CV-Amoxicillin prescriptions presented an opposite trend.

Indeed, the rate of CV-Amoxicillin prescriptions increased from $26.4 \%$ in 2010 to $29.2 \%$ in 2015 , while the frequency of amoxicillin use and the 'wait and see' approach showed a slight increase until 2014 followed by a decrease in 2015 . The frequency of other antibiotic use was constant over time (Fig. 2 with prescription rate provided in Table 2).

\section{Pharyngitis population}

Among 52,341 children, 126,098 distinct pharyngitis or tonsillitis diagnoses were recorded. $40.5 \%$ of them (51, $144 / 126098)$ were recorded as non-defined pharyngitis, $30.1 \%(37,929 / 126098)$ as GABHS pharyngitis, and $29.4 \%(37,025 / 126098)$ as non- GABHS pharyngitis. The mean age was 5 years (IQ 3-8), with $76 \%(95,972$ / 126098) of pharyngitis diagnosed after 3 years of age.

A rapid strep test was used in $34.8 \%$ of cases $(43,927 /$ $126098)$, having a positive result in $56.8 \%$ of samples (10, 299/18120 with $58.7 \%$ missing data regarding overall test results). Almost $86.2 \% \quad(32,700 / 37929)$ of GABHS pharyngitis cases were diagnosed using the rapid strep test, whereas most of the non- GABHS pharyngitis were 
Table 1 Distribution of first line antibiotic prescriptions for AOM with percentages referred to total prescription by age bands. Pedianet, Italy, 2010-2015

\begin{tabular}{|c|c|c|c|c|c|c|c|c|c|c|c|c|c|c|}
\hline & \multirow{2}{*}{\multicolumn{2}{|c|}{$\begin{array}{l}\text { Amoxicillin } \\
(N=15,906)\end{array}$}} & \multirow{2}{*}{\multicolumn{2}{|c|}{$\begin{array}{l}\text { CV- } \\
\text { Amoxicillin } \\
(N=14,865)\end{array}$}} & \multirow{2}{*}{\multicolumn{2}{|c|}{$\begin{array}{l}\text { Cephalosporins - III } \\
\text { gen. } \\
(N=9114)\end{array}$}} & \multirow{2}{*}{\multicolumn{2}{|c|}{$\begin{array}{l}\text { Cephalosporins - I/II } \\
\text { gen. } \\
(N=4056)\end{array}$}} & \multirow{2}{*}{\multicolumn{2}{|c|}{$\begin{array}{l}\text { Macrolides/ } \\
\text { Lincosamides } \\
(N=1347)\end{array}$}} & \multirow{2}{*}{\multicolumn{2}{|c|}{$\begin{array}{l}\text { Other }^{a} \\
\overline{(N=32)}\end{array}$}} & \multirow{2}{*}{\multicolumn{2}{|c|}{$\begin{array}{l}\text { Total } \\
(N=45,320)\end{array}$}} \\
\hline & & & & & & & & & & & & & & \\
\hline & $\bar{N}$ & (\%) & $\bar{N}$ & (\%) & $\bar{N}$ & (\%) & $\bar{N}$ & $(\%)$ & $\bar{N}$ & (\%) & $\bar{N}$ & (\%) & $\bar{N}$ & (\%) \\
\hline$\leq 6$ months & 486 & (54.1) & 259 & $(28.8)$ & 78 & (8.7) & 63 & $(7.0)$ & 13 & (1.4) & 0 & (0) & 899 & $(2.0)$ \\
\hline $6 \mathrm{mo} .<$ age $\leq 24 \mathrm{mo}$ & 4378 & (44.6) & 2630 & (26.8) & 1670 & $(17.0)$ & 945 & (9.6) & 186 & (1.9) & 14 & $(0.14)$ & 9823 & $(21.7)$ \\
\hline$>24$ months & 11,042 & (31.9) & 11,976 & $(34.6)$ & 7366 & $(21.3)$ & 3048 & (8.8) & 1148 & (3.3) & 18 & $(0.05)$ & 34,598 & $(76.3$ \\
\hline
\end{tabular}

${ }^{a}$ Clofoctol

diagnosed without using the test $(72.7 \%-26,923 / 37025)$ (data not shown, see Additional file 1).

Overall, in $63.1 \%(79,620 / 126098)$ of pharyngitis an antibiotic was prescribed, $20.7 \%(16,466 / 79620)$ for nonGABHS pharyngitis, $43.5 \%(34,671 / 79620)$ for GABHS pharyngitis and $35.8 \%(28,483 / 79620)$ for non-defined pharyngitis (data not shown, see Additional file 2).

The $94.7 \%$ of pharyngitis with a positive test result (9749/10299) received a prescription, while for cases with a negative test result just $8.2 \%$ received an antibiotic prescription (605/7415).

As regarding diagnosis not confirmed by the test, an antibiotic was prescribed in $90.2 \%$ of GABHS pharyngitis $(24,922 / 27630)$ and in $53.6 \%(15,861 / 29610)$ of nonGABHS pharyngitis.

Moreover, amoxicillin was prescribed in 55.8\% (5438/ 9749) of pharyngitis with a positive test result versus CV-Amoxicillin and cephalosporins of III generation prescribed in $24 \%(2341 / 9749)$ and $11.6 \%$ (1127/9749), respectively. The probability of having prescribed amoxicillin or CV-Amoxicillin with a diagnosis of GABHS pharyngitis not confirmed by the test was $32.1 \%$ (8004/ $24922)$ and $16.1 \%$ (4022/24922), respectively (Table 3).

Patients diagnosed with non- GABHS pharyngitis, regardless of the strep test, received CV-Amoxicillin in $30.6 \%(4856 / 15861)$ of cases and cephalosporins of II and III/IV generation in about $11.6 \%(1835 / 15861)$ and $28.1 \%(4458 / 15861)$ of the diagnosis; the rate of amoxicillin prescription alone for non- GABHS diagnosis not confirmed by the test was $18.6 \%(2944 / 15861)$ (Table 4). Distribution of first line treatment antibiotic therapy for non-defined pharyngitis is reported in Additional file 3.

\section{Discussion}

This study provides interesting insight into paediatric antibiotic prescriptions at the primary care level in Italy. Only a few reports on antibiotic prescriptions for pediatric populations have been published and even fewer linking antibiotic prescription to the diagnosis in primary care settings $[34,35]$.

In particular, the possibility to link antibiotic prescriptions with the related diagnosis in a longitudinal dataset is helpful for tracking prescribers' attitudes and understanding prescription trends over time.

In keeping with the literature, the pediatric population with AOM included in our study had a mean age of 34 years ( 44 months), with $22 \%$ children between 6 and 24 months and only $2 \%$ younger than 6 months of age.

Despite the mean age of the population analyzed the "wait and see" was adopted only in $18.5 \%$ of the cases as previously noted in other studies conducted in the hospital setting [36, 37].

However, we were unable to identify the use of delayed prescription approach where the physician provides an antimicrobial prescription at the time of

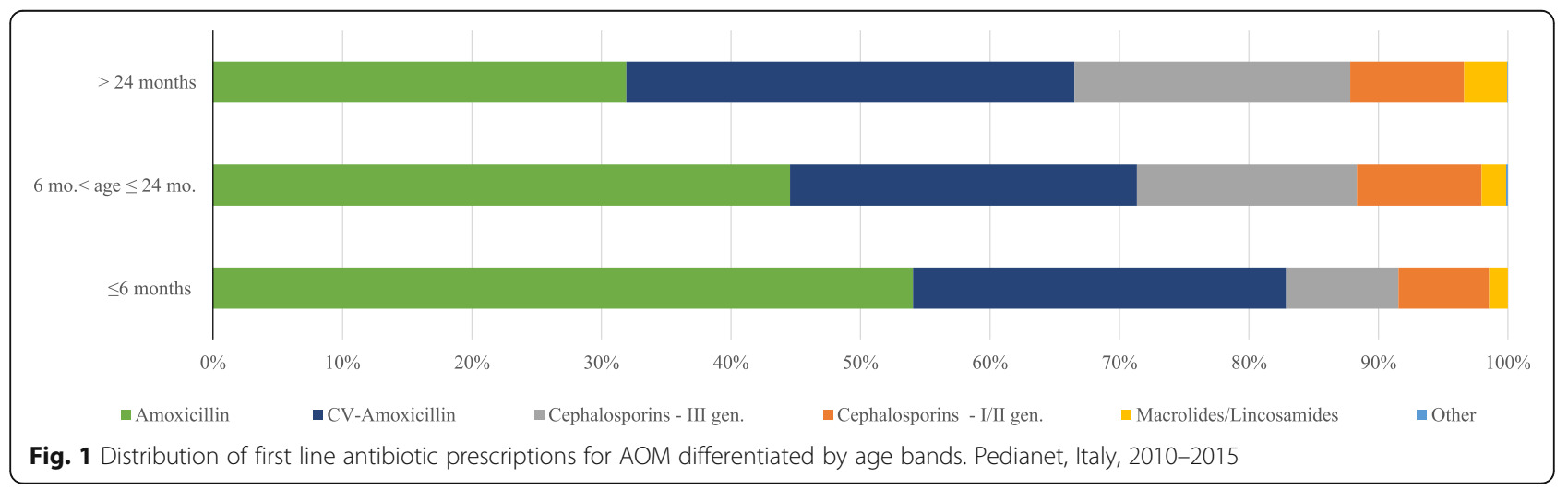




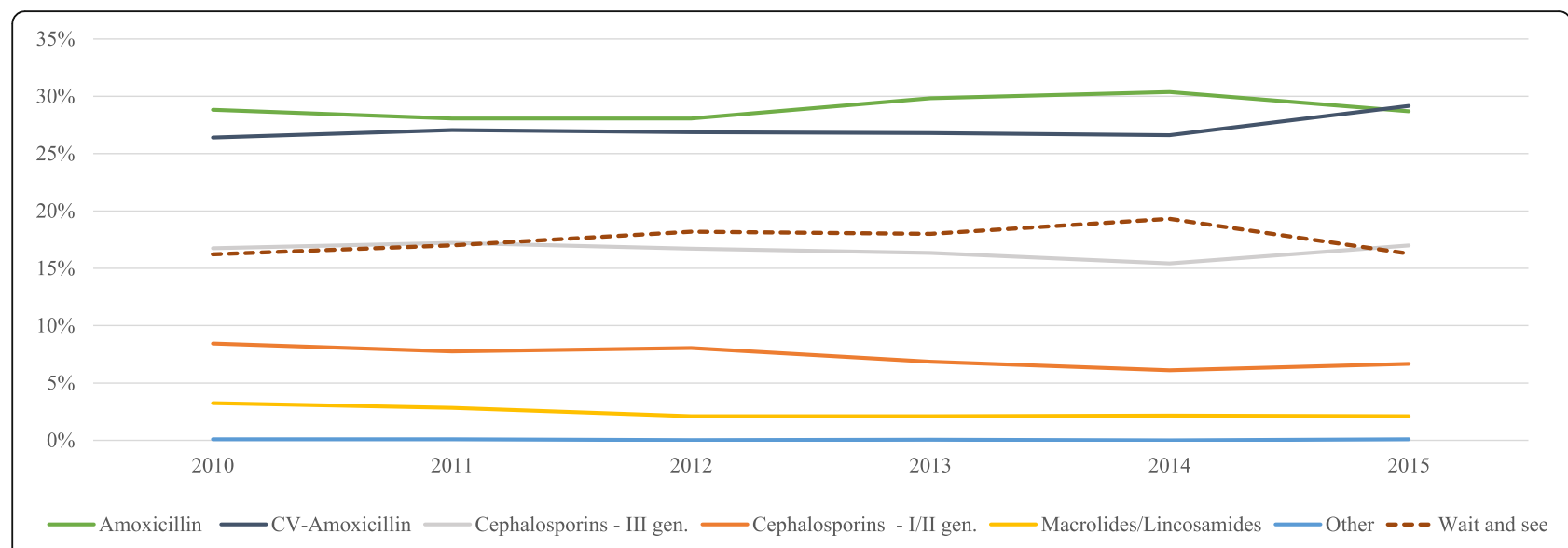

Fig. 2 Distribution of first line treatment approach for AOM by years. Pedianet, Italy, 2010-2015

diagnosis but asks parents to wait 48 to $72 \mathrm{~h}$ and administer the antibiotic only if there is no improvement in symptoms [29, 38].

An increase in broad spectrum prescriptions has been associated with the increase of children's age; more than half of the prescriptions for children older than 24 months in our study were for broad spectrum antibiotics. In contrast, more than $60 \%$ of children younger than 6 months received narrow spectrum antibiotics. This attitude may derive from the fear of beta lactamase producing bacterial infections for children over 24 months of age, possibly acquired in crowded places such nursery schools, or to the uncertainty of possible cumulative resistance in this age group after previous treatment with narrow-spectrum antibiotics. This prescribing attitude has also been reported in hospital settings and reflects prescription data from other pharmacoepidemiological studies [15].

The general trend towards broad spectrum antibiotic prescription as first line treatment was also reflected in the annual analysis where it was clear that at the end of the period analyzed (2015), after a previous slight increment in amoxicillin prescriptions, the guideline recommended first line approaches rate (together with 'wait and see' approach) decreased.

As regarding pharyngitis, our analysis found that the test was used mostly in GABHS diagnosis compared to non- GABHS ones, maybe due to the fact that pediatricians used this tool to confirm the empirical bacterial etiology instead of excluding it. This demonstrates apparent trust in clinical diagnosis by treating physician.

However, the fact that more antibiotics, mostly broadspectrum (CV-Amoxicillin and III gen. cephalosporin), were prescribed for a clinical diagnosis of GABHS pharyngitis indicates a more cautious interpretation of the clinical symptom evaluation.

Moreover, children with rapid test confirmed GABHS pharyngitis received mostly narrow-spectrum antibiotics (amoxicillin) while those without test results were equally likely to receive narrow or broad-spectrum treatment.

Almost 5\% of the children with rapid test positive GABHS pharyngitis were not treated with antibiotics. These results are comparable with the European

Table 2 Distribution of first line treatment approach for AOM with percentages referred to total treatments by years. Pedianet, Italy, 2010-2015

\begin{tabular}{|c|c|c|c|c|c|c|c|c|c|c|c|c|c|c|}
\hline & \multirow{2}{*}{\multicolumn{2}{|c|}{$\frac{\text { Amoxicillin }}{(N=15,906)}$}} & \multirow{2}{*}{\multicolumn{2}{|c|}{$\frac{\text { CV-Amoxicillin }}{(N=14,865)}$}} & \multirow{2}{*}{\multicolumn{2}{|c|}{$\frac{\text { Cephalosporins - III gen. }}{(N=9114)}$}} & \multirow{2}{*}{\multicolumn{2}{|c|}{$\frac{\text { Cephalosporins - I/II gen. }}{(N=4056)}$}} & \multirow{2}{*}{\multicolumn{2}{|c|}{$\frac{\text { Macrolides/ Lincosamides }}{(N=1347)}$}} & \multirow{2}{*}{\multicolumn{2}{|c|}{$\frac{\text { Other }^{a}}{(N=32)}$}} & \multirow{2}{*}{\multicolumn{2}{|c|}{$\begin{array}{l}\text { Wait and see } \\
(N=9623)\end{array}$}} \\
\hline & & & & & & & & & & & & & & \\
\hline & $\bar{N}$ & (\%) & $\bar{N}$ & (\%) & $\bar{N}$ & (\%) & $\mathrm{N}$ & (\%) & $\bar{N}$ & (\%) & $\bar{N}$ & (\%) & $\bar{N}$ & (\%) \\
\hline 2010 & 2868 & $(28.8)$ & 2627 & $(26.4)$ & 1667 & $(16.8)$ & 840 & (8.4) & 323 & (3.2) & 9 & $(0.1)$ & 1614 & $(16.2)$ \\
\hline 2011 & 2840 & (28.1) & 2738 & (27.1) & 1742 & $(17.2)$ & 785 & (7.8) & 287 & (2.8) & 9 & (0.1) & 1720 & (17.0) \\
\hline 2012 & 2748 & (28.1) & 2630 & (26.9) & 1636 & $(16.7)$ & 787 & (8.0) & 205 & $(2.1)$ & 2 & (0) & 1782 & $(18.2)$ \\
\hline 2013 & 2902 & (29.8) & 2606 & (26.8) & 1590 & $(16.3)$ & 666 & (6.8) & 205 & $(2.1)$ & 6 & (0.1) & 1752 & (18.0) \\
\hline 2014 & 2549 & (30.4) & 2233 & (26.6) & 1295 & $(15.4)$ & 513 & $(6.1)$ & 181 & $(2.2)$ & 0 & (0) & 1621 & (19.3) \\
\hline 2015 & 1999 & (28.7) & 2031 & $(29.2)$ & 1184 & $(17.0)$ & 465 & (6.7) & 146 & $(2.1)$ & 6 & (0.1) & 1134 & (16.3) \\
\hline
\end{tabular}

${ }^{\mathrm{a} C l o f o c t o l}$ 
Table 3 Distribution of first line treatment antibiotic therapy for GABHS pharyngitis. Pedianet, Italy, 2010-2015

\begin{tabular}{|c|c|c|c|c|c|c|}
\hline & \multicolumn{6}{|c|}{ GABHS pharyngitis } \\
\hline & \multicolumn{6}{|c|}{$(N=37,929)$} \\
\hline & \multicolumn{2}{|c|}{ No test- No result/Dubious result } & \multicolumn{2}{|c|}{ Positive test } & \multicolumn{2}{|l|}{ Total } \\
\hline & $\bar{N}$ & (\%) & $\bar{N}$ & (\%) & $\mathrm{N}$ & (\%) \\
\hline Amoxicillin & 10,602 & $(42.5)$ & 5438 & $(55.8)$ & 16,040 & $(46.3)$ \\
\hline CV-Amoxicillin & 8004 & (32.1) & 2341 & [24] & 10,345 & (29.8) \\
\hline Cephalosporins - III gen. & 4022 & $(16.1)$ & 1127 & (11.6) & 5149 & $(14.9)$ \\
\hline Cephalosporins - II gen. & 1349 & $(5.4)$ & 389 & [4] & 1738 & [5] \\
\hline Macrolides/Lincosamides & 925 & (3.7) & 449 & (4.6) & 1374 & [4] \\
\hline Other ${ }^{a}$ & 20 & $(0.1)$ & 5 & $(0.1)$ & 25 & $(0.1)$ \\
\hline Total treated & 24,922 & $(90.2)$ & 9749 & $(94.7)$ & 34,671 & (91.4) \\
\hline Total not treated & 2708 & (9.8) & 550 & (5.3) & 3258 & (8.6) \\
\hline
\end{tabular}

${ }^{\mathrm{a} C l o f o c t o l}$

population of pediatric GABHS carriers found in the literature [32].

These results seemed to confirm once again that diagnostic uncertainty is one of the determinants for overall antibiotic over prescription [39], together with perceived parental expectations of an antibiotic prescription and fear of under treatment as previously found in other studies [40].

Focusing more deeply on the antibiotics prescribed it is worrisome realizing that III generation cephalosporins are so highly prescribed for non- GABHS pharyngitis whether or not confirmed by the test $(22 \%$ vs $28 \%$ of total prescriptions for not confirmed and confirmed, respectively). These outcomes seemed to reflect a trend already known in the hospital setting [14] both in pediatric and adult populations [41], confirming an abuse of these antibiotics. However, it should be considered that, despite the initial pharyngitis diagnosis, the pediatrician could have prescribed an antibiotic suspecting another upper or lower respiratory tract infection.

Another analysis conducted by de Bie et al. [42], confirmed the overuse of antibiotics in the Italian pediatric population, compared especially with north European countries; even though amoxicillin remains the most prescribed antibiotic, the prevalence of broad spectrum prescriptions in Italy was almost double compared to the United Kingdom and the Netherlands where amoxicillin prescriptions accounted for $50 \%$ of antibiotic prescriptions. These results are in line with our findings especially for $\mathrm{AOM}$ and non-GABHS pharyngitis. Furthermore, an 8 -year survey conducted in all hospitals in the EmiliaRomagna Region showed a steadily increasing consumption of broad-spectrum antibiotics as noted in our study for $\mathrm{AOM}$ prescriptions and a considerable decrease of narrow-spectrum ones [43].

Table 4 Distribution of first line treatment antibiotic therapy for non-GABHS pharyngitis. Pedianet, Italy, 2010-2015

\begin{tabular}{|c|c|c|c|c|c|c|}
\hline & \multicolumn{6}{|c|}{ non-GABHS pharyngitis } \\
\hline & \multicolumn{6}{|c|}{$(N=37,025)$} \\
\hline & \multicolumn{2}{|c|}{ No test- No result/Dubious result } & \multicolumn{2}{|c|}{ Negative test } & \multicolumn{2}{|l|}{ Total } \\
\hline & $\mathrm{N}$ & $(\%)$ & $\mathrm{N}$ & $(\%)$ & $\mathrm{N}$ & $(\%)$ \\
\hline Amoxicillin & 2944 & (18.6) & 221 & $(36.5)$ & 3165 & $(19.2)$ \\
\hline CV-Amoxicillin & 4856 & (30.6) & 149 & (24.6) & 5005 & $(30.4)$ \\
\hline Cephalosporins - III gen. & 4458 & $(28.1)$ & 100 & $(16.5)$ & 4558 & $(27.7)$ \\
\hline Cephalosporins - II gen. & 1835 & $(11.6)$ & 28 & $(4.6)$ & 1863 & $(11.3)$ \\
\hline Macrolides/Lincosamides & 1652 & $(10.4)$ & 99 & $(16.4)$ & 1751 & $(10.6)$ \\
\hline Other $^{\mathrm{a}}$ & 116 & $(0.7)$ & 8 & $(1.3)$ & 124 & $(0.8)$ \\
\hline Total treated & 15,861 & (53.6) & 605 & $(8.2)$ & 16,466 & $(44.5)$ \\
\hline Total not treated & 13,749 & (46.4) & 6810 & (91.8) & 20,559 & (55.5) \\
\hline
\end{tabular}


As for the outpatient population, according to drug prescriptions dispensed during 2006 by some retail pharmacies in Italy, $52 \%$ of the paediatric population received at least one antibiotic therapy, a little bit more for males and less for females [4]. It has been estimated that almost half of antibiotics prescribed by a primary care physician are unnecessary [44] since the majority of them are prescribed for common pediatric infections such as pharyngitis (considered also in our analysis) that mainly have a viral cause.

For the aforementioned reasons it is imperative to find a way to reduce antibiotics use, particularly in pediatric populations.

As regarding $\mathrm{AOM}$ and pharyngitis few regions implemented guidelines for the diagnosis and the treatment of these illnesses (e.g. Veneto region implemented the "Cure Primarie" project in 2006) and some consensus and guidelines written both by pediatric ENT specialist and pediatric infectious diseases specialist have been published in the last decades [22, 23, 30].

As our results point out, guidelines do not seem to be the most suitable tool in primary care settings where the need of rapid decision making limit the consultation of lengthy guidelines.

Interestingly, Gerber et al. [45] proved that clinician education coupled with audit and feedback of prescribing significantly improved antibiotic use for outpatient children; the overall proportion of broad-spectrum antibiotic prescriptions decreased from 26.8 to $14.3 \%$ after the intervention. Although very effective, these two core antibiotic stewardship (AS) interventions are expensive (median cost \$187.400) [46] and this could limit their implementation in the Italian healthcare setting.

On the other hand, other AS interventions such as clinical pathways $(\mathrm{CP})$ could represent useful and practical evidence-based tools to guide antibiotic prescribing where both personnel and economic resources are restricted. In Italy clinical pathways for pharyngitis, AOM and community acquired pneumonia were successfully implemented in a tertiary care hospital with a significant reduction of broad spectrum antibiotic prescriptions and of total antibiotic cost [17, 47].

The Pedianet database or other similar data sources could be valuable tools to measure the effectiveness and costs of AS interventions as well as other healthcare policy actions. The possibility of accessing data related to the daily activities of FP is a unique resource, both for studying individual diseases, as well for pharmacoepidemiological and pharmacoeconomical analysis. Pedianet is an example of an efficient pediatric outpatient network collecting specific data from computerized clinical files. With more than 300 Italian pediatricians enrolled throughout the country, this network has been proven to be able to carry out epidemiological studies as well as pharmacovigilance ones [48-50].

Our study had several limitations including the retrospective nature of the analysis and the lack of manual evaluation and validation of the diagnosis potentially including false positive cases in the analysis.

Secondly, as mentioned earlier the fact that we cannot identify the delayed prescription for AOM is one limitation.

As regarding rapid strep usage not all clinicians have the possibility to perform the test in their practice, thus this could represent a bias.

Finally, with our data it was not possible to separate pediatricians who might have received an educational training on treatment of the diagnosis considered that may have changed their practice through the years. On the other hand, the high number of FP enrolled and the variety of settings of their practices (urban, suburban, rural) is a positive element in supporting the generalizability of results.

\section{Conclusion}

The analysis conducted using a large Italian family pediatricians' database confirmed the increasing prescriptions rate of broad-spectrum antibiotics (especially amoxicillin/ clavulanate and III generation cephalosporins) for AOM and pharyngitis already observed in the literature. With the increasing concern about antimicrobial resistance, the identification of potential barriers for successful intervention remains crucial, especially where resources are limited. Future studies should examine the best approach to guide pediatric antibiotic prescribing in Italian outpatient settings where challenges are represented mainly by high rates of patient turnover and rapid decisionmaking, usually without microbiology support.

\section{Additional files}

Additional file 1: Distribution of test results according to pharyngitis diagnosis. Pedianet, Italy, 2010-2015. (XLSX 9 kb)

Additional file 2: Distribution of first line approach according to pharyngitis diagnosis. Pedianet, Italy, 2010-2015. (XLSX 9 kb)

Additional file 3: Distribution of first line treatment antibiotic therapy for non-defined pharyngitis. Pedianet, Italy, 2010-2015. (XLSX 9 kb)

\section{Abbreviation \\ AOM: Acute Otitis Media; AS: Antibiotic Stewardship; CP: Clinical Pathways; CV-Amoxicillin: Amoxicillin and Clavulanic Acid; ENT: Ear Nose and Throat; FIMP: Federazione Italiana Medici Pediatri; FP: Family Pediatrician; GABHS: Group A $\beta$-hemolytic streptococcus; ICD-9: 9th International Statistical Classification of Diseases and Related Health Problems system; IQ: Inter- Quartile; SIP: Società Italiana di Pediatria}

\section{Acknowledgments}

The authors thank all the family pediatricians collaborating in Pedianet. Angelo Alongi, Roberta Angelini, Giovanni Avarello, Lucia Azzoni, Maria Carolina Barbazza, Maria Barberi Frandanisa, Patrizia Barbieri, Gabriele Belluzzi, Eleonora Benetti, Roberto Bezzi, Franca Boe, Stefano Bollettini, Andrea Bruna, Ivana Brusaterra, Roberto Budassi, Massimo Caccini, Laura Cantalupi, Luigi 
Cantarutti, Luigia Caprio, Massimo Castaldo, Stefano Castelli, Serenella Castronuovo, Monica Cavedagni, Stefania Censini, Giuseppe Egidio Cera, Carla Ciscato, Mariangela Clerici Schoeller, Giuseppe Collacciani, Fabrizio Comaita, Ugo Alfredo Conte, Nicola Costanzo, Sandra Cozzani, Giancarlo Cuboni, Vito Francesco D'Amanti, Rita De Angelis, Roberto De Clara, Annamaria De Marchi, Emanuele De Nicolò, Gian Piero Del Bono, Gigliola Del Ponte, Tiziana Di Giampietro, Giuseppe Di Mauro, Giuseppe Di Santo, Piero Di Saverio, Marco Dolci, Mattia Doria, Stefano Drago, Pietro Falco, Mario Fama, Marco Faraci, Tania Favilli, Mariagrazia Federico, Michele Felice, Enrico Ferrara, Marta Ferrarese, Michele Ferretti, Paolo Forcina, Claudio Frattini, Ezio Frison, Fabrizio Fusco, Giovanni Gallo, Andrea Galvagno, Alberta Gentili, Pierfrancesco Gentilucci, Giuliana Giampaolo, Giuseppe Giancola, Silvia Girotto, Costantino Gobbi, Mauro Grelloni, Mirco Grugnetti, Urania Lagrasta, Massimo Landi, Paola Lasalvia, M.Rosaria Letta, Giuseppe Lietti, Cinzia Lista, Ricciardo Lucantonio, Francesco Luise, Diego Luotti, Nadia Macropodio, Francesca Marine, Lorenzo Mariniello, Gabriele Marostica, Sergio Masotti, Stefano Meneghetti, Massimo Milani, Stella Vittoria Milone, Angela Maria Monteleone, Pierangela Mussinu, Carmen Muzzolini, Flavia Nicoloso, Laura Olimpi, Maria Maddalena Palma, Vittorio Pandolfini, Angela Pasinato, Andrea Passarella, Pasquale Pazzola, Monica Perin, Danilo Perri, Silvana Pescosolido, Giovanni Petrazzuoli, Giuseppe Petrotto, Patrizia Picco, Ambrogina Pirola, Lorena Pisanello, Daniele Pittarello, Eleonora Polidoro, Elena Porro, Adolfo Francesco Porto, Elisabetta Profumo, Antonino Puma, Ferdinando Ragazzon, Paolo Rosas, Rino Rosignoli, Mariagiulia Rosina, Mariella Rossitto, Bruno Ruffato, Lucia Ruggieri, Annamaria Ruscitti, Annarita Russo, Pietro Salamone, Daniela Sambugaro, Luigi Saretta, Vittoria Sarno, Nico Maria Sciolla, Paolo Senesi, Carla Silvan, Valter Spanevello, Francesco Speranza, Maura Sticco, Francesco Storelli, Gianni Tamassia, Paolo Tambaro, Giacomo Toffol, Marco Tondello, Gabriele Tonelli, Angelo Tummarello, Sergio Venditti, Concetta Volpe, Francescopaolo Volpe, Aldo Vozzi. Finally the authors would like to acknowledge PENTA foundation.

\section{Authors' contributions}

$\mathrm{EB}$ has been involved in analysis and interpretation of data and in drafting and revising the manuscript. AC has been involved in the analysis of data and in drafting and revising the manuscript. DD has made substantial contributions to conception and design and has been deeply involved in drafting and revising critically the manuscript. RL and GC have been involved in revising the manuscript. LC and AS have made substantial contributions to conception and design, and acquisition of data, and analysis and interpretation of the data. CG has made substantial contributions to conception and design, and acquisition of data, and analysis and interpretation of the data and has been involved in revising the manuscript. All authors read and approved the final manuscript.

\section{Funding}

This work was supported in part by the Italian Ministry of University and Research under the PRIN Project (PROGETTI DI RICERCA DI RILEVANTE INTERESSE NAZIONALE - Bando 2017) - Prot. 2017728JPK.

\section{Availability of data and materials}

The datasets generated during and/or analyzed during the current study are available from the corresponding author on reasonable request.

\section{Ethics approval and consent to participate}

The study and the access to the database were approved by the Internal Scientific Committee.

\section{Consent for publication}

Not applicable.

\section{Competing interests}

The authors declare that they have no competing interests.

\section{Author details}

'Department for Woman and Child Health, University of Padua, Padua, Italy. ${ }^{2}$ Division of Paediatric Infectious Diseases, Department for Woman and Child Health, University of Padua, Via Giustiniani 3, 35141 Padua, Italy. ${ }^{3}$ PENTA Foundation, Padua, Italy. ${ }^{4}$ National Centre for Healthcare Research and Pharmacoepidemiology, University of Milano-Bicocca, Milan, Italy. ${ }^{5}$ Department of Statistics and Quantitative Methods, Unit of Biostatistics
Epidemiology and Public Health, University of Milano-Bicocca, Milan, Italy. ${ }^{6}$ Pedianet Project, Padua, Italy.

\section{Received: 11 February 2019 Accepted: 6 August 2019 \\ Published online: 17 August 2019}

\section{References}

1. van der Meer JW, Gyssens IC. Quality of antimicrobial drug prescription in hospital. Clin Microbiol Infect. 2001;7:12-5.

2. Gerber JS, et al. Variability in antibiotic use at Children's hospitals. Pediatrics. 2010;126:1067-73

3. Ashiru-Oredope D, et al. Antimicrobial stewardship: English surveillance Programme for antimicrobial utilization and resistance (ESPAUR). J Antimicrob Chemother. 2013;68:2421-3.

4. Clavenna A, et al. Drug prescriptions to outpatient children: a review of the literature. Eur J Clin Pharmacol. 2009;65:749-55.

5. van Houten MA, et al. Antibiotic utilisation for hospitalised paediatric patients. Int J Antimicrob Agents. 1998;10:161-4.

6. Potocki $M$, et al. Prospective survey of antibiotic utilization in pediatric hospitalized patients to identify targets for improvement of prescription. Infection. 2003;31(6):398-403.

7. Hajdu A, et al. A point prevalence survey of hospital-acquired infections and antimicrobial use in a paediatric hospital in North-Western Russia. J Hosp Infect. 2007:66:378-84.

8. Berild D, et al. A controlled intervention study to improve antibiotic use in a Russian paediatric hospital. Int J Antimicrob Agents. 2008;31:478-83.

9. Ang $L$, et al. A point prevalence study of infection and antimicrobial use at a UK children's hospital. J. Hosp. Infect. 2008;68:372-4.

10. Nyquist $A C$, et al. Antibiotic prescribing for children with colds, upper respiratory tract infections, and bronchitis. J Am Med Assoc. 1998;279:875-7.

11. Hecker MT, et al. Unnecessary use of antimicrobials in hospitalized patients. Arch Intern Med. 2003;163:972.

12. El Sayed MF, et al. Prospective study on antibiotics misuse among infants with upper respiratory infections. Eur J Pediatr. 2009;168:667-72.

13. Faringotonsillite in età pediatrica. Guida rapida 2015 - E-R Agenzia sanitaria e sociale regionale. (https://assr.regione.emilia-romagna.it/it/servizi/ pubblicazioni/rapporti-documenti/faringotonsillite-guida-rapida-2015). (Accessed 18 Dec 2018).

14. De Luca $M$, et al. Antibiotic prescriptions and prophylaxis in Italian children. Is it time to change? Data from the ARPEC project. PLoS One. 2016;11:1-14.

15. Piovani $D$, et al. Assessing the quality of paediatric antibiotic prescribing by community paediatricians: a database analysis of prescribing in Lombardy. BMJ Paediatr open. 2017;1:e000169.

16. Palma $\mathrm{S}$, et al. The impact of the Italian guidelines on antibiotic prescription practices for acute otitis media in a paediatric emergency setting. Ital J Pediatr. 2015;41:4-9.

17. Dona $D$, et al. The impact of clinical pathways on antibiotic prescribing for acute otitis media and pharyngitis in the emergency department. Pediatr Infect Dis J. 2018;37:1.

18. Marchisio P, et al. Patterns in acute otitis media drug prescriptions: a survey of Italian pediatricians and otolaryngologists. Expert Rev Anti-Infect Ther. 2014;12:1159-63.

19. Bluestone CD, et al. Ten-year review of otitis media pathogens. Pediatr Infect Dis J. 1992;11:S7-11.

20. Marchisio P, et al. Burden of acute otitis media in primary care pediatrics in Italy: a secondary data analysis from the Pedianet database. BMC Pediatr. 2012;12:705.

21. Monasta $L$, et al. Burden of disease caused by otitis media: systematic review and global estimates. PLoS One. 2012;7:e36226.

22. Marchisio, P. et al. Otite Media Acuta: dalla diagnosi alla prevenzione Linee Guida della Società Italiana di Pediatria. (2010)

23. L'utilizzo giudizioso della terapia antibiotica nel trattamento delle patologie infettive in età evolutiva. - FIMP ( https://www.pacinimedicina.it/wpcontent/uploads/RivFIMP_3_16-1.pdf) (2016).

24. Shaikh $\mathrm{N}$, et al. Prevalence of streptococcal pharyngitis and streptococcal carriage in children: a meta-analysis e564. Pediatrics. 2010;126:557.

25. Regoli $M$, et al. Update on the management of acute pharyngitis in children. Ital J Pediatr. 2011. https://doi.org/10.1186/1824-7288-37-10.

26. Kronman MP, et al. Bacterial prevalence and antimicrobial prescribing trends for acute respiratory tract infections. Pediatrics. 2014;134:e956-65. 
27. Fierro $\mathrm{J}$, et al. Variability in the diagnosis and treatment of group a streptococcal pharyngitis by primary care pediatricians. Infect Control Hosp Epidemiol. 2014;35:S79-85.

28. Management of sore throat and indications for tonsillectomy. A national clinical guideline. Edinburgh: Scottish intercollegiate guidelines network (SIGN); methodology; 2010. https://www.sign.ac.uk/assets/sign117.pdf

29. Respiratory tract infections (self-limiting): prescribing antibiotics. NICE; Clinical guideline. 2008. (https://www.nice.org.uk/guidance/cg69/resources/respiratorytract-infections-selflimiting-prescribing-antibiotics-pdf-975576354757.

30. Linee Guida Italiane per la gestione della faringotonsillite in età pediatrica: sintesi e commento. SIPPS- Società Italiana di Pediatria Preventiva e Sociale. 2012(https://www.sipps.it/pdf/lineeguida/faringotonsillite.pdf).

31. Systemic antibiotic treatment in upper and lower respiratory tract infections: official French guidelines. Agence Française de Sécurité Sanitaire des Produits de Santé. Clin Microbiol Infect. 2003;9:1162-78.

32. Shaikh $\mathrm{S}$, et al. Role of rapid diagnostic tests for guiding outpatient treatment of febrile illness in Liaquat University hospital. Pak J Med Sci. 2013;29:1167-72.

33. Corbeel L. The 'wait and see' approach of acute otitis media. Eur J Pediatr. 2005;164:1-2.

34. Rossignoli A, et al. Antibiotic prescription and prevalence rate in the outpatient paediatric population: analysis of surveys published during 20002005. Eur J Clin Pharmacol. 2007;63:1099-106.

35. Clavenna A, et al. Differences in antibiotic prescribing in paediatric outpatients. Arch Dis Child. 2011;96:590-5.

36. Fischer $\mathrm{T}$, et al. National Trends in emergency department antibiotic prescribing for children with acute otitis media, 1996-2005. Acad Emerg Med. 2007;14:1172-5.

37. Cates CJ. Delayed antibiotics for children with acute otitis media: is practice change sustainable? Evid Based Nursing. 2009;12:39-40.

38. Little P. Pragmatic randomised controlled trial of two prescribing strategies for childhood acute otitis media. BMJ. 2001;322:336-42.

39. Pichichero ME. Understanding antibiotic overuse for respiratory tract infections in children. Pediatrics. 1999;104(6):1384-8.

40. Moro ML, et al. Why do paediatricians prescribe antibiotics? Results of an Italian regional project. BMC Pediatr. 2009;9:69.

41. Vaccheri $A$, et al. A 3 year survey on the use of antibacterial agents in five Italian hospitals. J Antimicrob Chemother. 2008;61:953-8.

42. De Bie $\mathrm{S}$, et al. Using prescription patterns in primary care to derive new quality indicators for childhood community antibiotic prescribing. Pediatr Infect Dis J. 2016;35:1317-23.

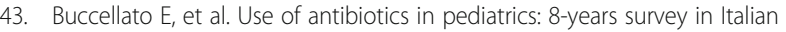
hospitals. PLoS One. 2015;10(9):e0139097.

44. Pichichero ME. Dynamics of antibiotic prescribing for children. J Am Med Assoc. 2002;287:3133-5.

45. Gerber JS, et al. Effect of an outpatient antimicrobial stewardship intervention on broad-spectrum antibiotic prescribing by primary care pediatricians: a randomized trial. J Am Med Assoc. 2013;309:2345-52.

46. Zachariah $P$, et al. Costs of antimicrobial stewardship programs at US Children's hospitals. Infect Control Hosp Epidemiol. 2016:37:852-4.

47. Donà $\mathrm{D}$, et al. Effects of clinical pathway implementation on antibiotic prescriptions for pediatric community-acquired pneumonia. PLoS One. 2018; 13:e0193581.

48. Donà $\mathrm{D}$, et al. Community-acquired rotavirus gastroenteritis compared with adenovirus and norovirus gastroenteritis in Italian children: a Pedianet study. Int J Pediatr. 2016;2016:1-10.

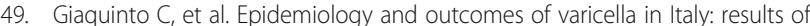
a prospective study of children (0-14 years old) followed up by pediatricians (Pedianet study). Ann Ig. 2002;14(4 Suppl 6):21-7. Italian.

50. Schuemie MJ, et al. Replication of the OMOP experiment in europe: evaluating methods for risk identification in electronic health record databases. Drug Saf. 2013. https://doi.org/10.1007/s40264-013-0109-8.

\section{Publisher's Note}

Springer Nature remains neutral with regard to jurisdictional claims in published maps and institutional affiliations.

\section{Ready to submit your research? Choose BMC and benefit from:}

- fast, convenient online submission

- thorough peer review by experienced researchers in your field

- rapid publication on acceptance

- support for research data, including large and complex data types

- gold Open Access which fosters wider collaboration and increased citations

- maximum visibility for your research: over $100 \mathrm{M}$ website views per year

At $\mathrm{BMC}$, research is always in progress.

Learn more biomedcentral.com/submissions 